\title{
USO DA ANÁLISE MULTIVARIADA PARA SUBSIDIAR NO MONITORAMENTO DA QUALIDADE DA ÁGUA SUBTERRÂNEA NA BACIA SEDIMENTAR DO ARARIPE, SUL DO ESTADO DO CEARÁ
}

\author{
USE OF MULTIVARIATE ANALYSIS TO SUBSIDIZE MONITORING \\ THE QUALITY OF GROUNDWATER IN THE ARARIPE SEDIMENTARY BASIN, SOUTH OF \\ THE STATE OF CEARÁ
}

\author{
Introdução \\ Materiais e métodos \\ Resultados e discussões \\ Considerações finais \\ Referências
}

Maria da Conceição Rabelo GOMES, Raimunda Moreira da FRANCA

Universidade Federal do Ceará. Av. da Universidade, 2853 - Benfica, Fortaleza, CEP 60020-181

Emails: conceicaorabelo@yahoo.com.br; francacariri@yahoo.com.br

\begin{abstract}
RESUMO - O monitoramento das águas subterrâneas geralmente envolve a determinação de muitas variáveis para caracterizar a qualidade da água, as quais são fortemente correlacionadas entre si, dificultando o entendimento de suas inter-relações. Com a utilização de análise multivariada é possível reduzir o número de variáveis, indicar as que são responsáveis pela dispersão das observações e evidenciar agrupamentos, objeto deste estudo para o município de Juazeiro do Norte, Ceará. A partir destes resultados pode-se observar que as características das águas estão fortemente relacionadas com a formação geológica dos poços estudados. Portanto, medidas de monitoramento podem ser realizadas com base nos elementos hidroquímicos determinantes da qualidade das águas (CE, STD, dureza total, $\mathrm{Ca}^{2+}, \mathrm{Mg}^{2+}, \mathrm{K}^{+}, \mathrm{Cl}^{-}, \mathrm{pH}, \mathrm{HCO}_{3}$ e N-NO ) identificados pela análise fatorial. Estas medidas podem ser voltadas para conservação dos solos e melhor uso das águas subterrâneas do município de Juazeiro do Norte. Procedimentos de agrupar as amostras de águas similares em função das variáveis que definem a qualidade das águas facilitam o processo de gestão de recursos hídricos, principalmente nas atividades de monitoramento e de utilização das águas.

Palavras-chave: Poços, Hidroquímica, Análise fatorial, Análise de agrupamento.
\end{abstract}

\begin{abstract}
Groundwater monitoring usually involves the determination of many variables to characterize water quality, which are strongly correlated with each other, making it difficult to understand their interrelationships. With the use of multivariate analysis it is possible to reduce the number of variables, to indicate the ones that are responsible for the dispersion of the observations and to show groupings, object of this study for the city of Juazeiro do Norte, Ceará. From these results it can be observed that the water characteristics are strongly related to the geological formation of the studied wells. Therefore, monitoring measures can be performed based on the hydrochemical determinants of water quality (EC, TDS, total hardness, $\mathrm{Ca}^{2+}, \mathrm{Mg}^{2+}, \mathrm{K}^{+}, \mathrm{Cl}^{-}, \mathrm{pH}, \mathrm{HCO}_{3}$ and N-NO${ }_{3}$ ) identified by the factorial analysis. These measures can be focused on soil conservation and better use of groundwater in the municipality of Juazeiro do Norte. Procedures for grouping samples of similar waters according to variables that define water quality facilitate the process of water resources management, mainly in the activities of monitoring and water use. Keywords: Wells, Hydrochemistry, Factor analysis, Cluster analysis.
\end{abstract}

\section{INTRODUÇÃO}

A maior reserva de água subterrânea do estado do Ceará está localizada na Bacia Sedimentar do Araripe, porção sul do estado, na divisa com os estados de Pernambuco e Piauí. O relevo é constituído por dois domínios principais, planalto e depressão, conhecidos como Chapada do Araripe e Vale do Cariri, respectivamente (COGERH, 2009). A Chapada ocupa 73\% da Bacia Sedimentar do Araripe e compreende altitudes que variam de $1.000 \mathrm{~m}$ ao norte de Porteiras-CE, na porção leste, a $700 \mathrm{~m}$ nas imediações de Araripina-PE, na porção oeste (DNPM, 1996 apud COGERH, 2009), destacando-se na paisagem nordestina devido os desníveis em torno de $400 \mathrm{~m}$ com a superfície sertaneja que circunda a chapada.

Em estudos de avaliação e monitoramento da qualidade das águas subterrâneas, normalmente é utilizado muitas variáveis para sua caracterização e estas, por sua vez, são fortemente correlacionadas, dificultando o entendimento de suas inter-relações. Com a utilização de técnicas de análise multivariada é possível reduzir o número de variáveis, definir suas relações, identificar as que são responsáveis pela dispersão das observações e evidenciar agrupamentos (Brito et al., 2004). A análise multivariada auxilia na definição de quais variáveis são mais 
relevantes para a gestão hídrica, auxiliando na seleção de variáveis a partir de critérios mais objetivos. Estas técnicas têm sido empregadas em diferentes áreas. No contexto dos recursos hídricos subterrâneos Brito et al. (2006), Cloutier et al. (2008), Fernandes et al. (2010), Salgado et al. (2011), Gomes \& Cavalcante (2017), entre outros, utilizaram essa ferramenta estatística como suporte ao monitoramento e à gestão qualitativa da água subterrânea, principalmente em regiões semiáridas, que são de alta vulnerabilidade socioambiental. Assim, o objetivo desta pesquisa foi aplicar a análise multivariada para subsidiar no monitoramento da qualidade da água subterrânea no município de Juazeiro do Norte (porção leste da Bacia Sedimentar do Araripe).

Juazeiro do Norte é a maior cidade do interior cearense em aspecto de desenvolvimento, com população de 268.248 mil habitantes (densidade de $1.005 \mathrm{hab} / \mathrm{Km}^{2}$ ), com taxa de urbanização de 95,3\% (IBGE, 2010). Nesta área os recursos hídricos subterrâneos são a única fonte de abastecimento público e privado e a superexplotação já é evidenciada na intermitência da antiga rede de drenagem perene.

\section{MATERIAIS E MÉTODOS}

A pesquisa foi realizada na porção leste da Bacia Sedimentar do Araripe no Vale do Cariri, envolvendo parte do município de Juazeiro do Norte (9197770 - 9207000 N e 462000 - 471000 S, Zona 24S) (Figura 1), caracterizada por clima tropical quente semiárido, com temperatura média de 24 a $26{ }^{\circ} \mathrm{C}$ e precipitação pluvial média anual de $925 \mathrm{~mm}$, com período chuvoso de janeiro a maio
(IPECE, 2015) e uma área de $78 \mathrm{~km}^{2}$. A área é constituída por rochas do embasamento précambriano e rochas sedimentares pertencentes à Bacia Sedimentar do Araripe (CPRM, 2000). As rochas sedimentares são formadas do topo para a base, por depósitos aluvionares, coberturas arenosas, Formação Rio da Batateira, Formação Brejo Santo e a Formação Mauriti (Figura 1).

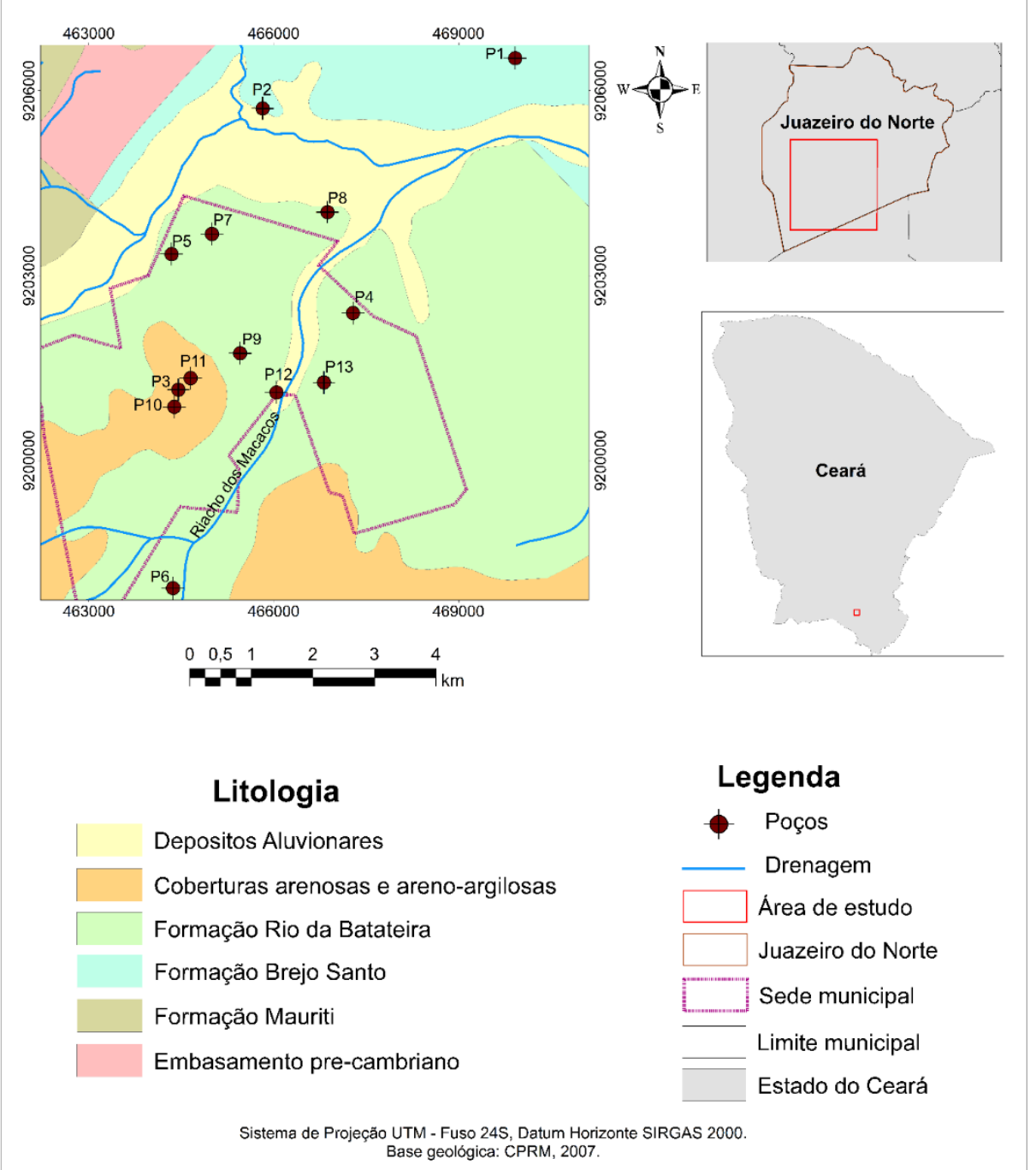

Figura 1 - Localização da área de estudo e poços analisados. 
$\mathrm{Na}$ área aflora e predomina a Formação Rio da Batateira, caracterizada por arenitos argilosos médios a finos, com espessura média de $200 \mathrm{~m}$, associado com a presença de depósitos aluvionares.

Esta formação constitui o aquífero livre explotado mais importante, sendo afetado pela ausência de controle na exploração dos poços e a precariedade de saneamento básico.
O estudo foi desenvolvido a partir de vinte e seis (26) análises físico-químicas de águas subterrâneas de Juazeiro do Norte (Figura 1 e tabelas 1 e 2) coletadas entre os períodos de chuva (janeiro) e seco (julho) de 2011 oriundas do banco de dados da Companhia de Gestão dos Recursos Hídricos $(\mathrm{COGERH})$. Esses dados foram processados no software SPSS Statistics, versão 17.0.

Tabela 1 - Análises físico-químicas das águas subterrâneas de Juazeiro do Norte/CE (janeiro/2011).

\begin{tabular}{|c|c|c|c|c|c|c|c|c|c|}
\hline \multicolumn{10}{|c|}{ Período de chuva (janeiro/2011) } \\
\hline Poços & pH & $\mathbf{C E}$ & Turbidez & Cor & STD & DT & $\mathbf{C a}$ & Mg & $\mathbf{N a}$ \\
\hline P1 & 5,80 & 610,00 & 0,50 & 0,00 & 509,70 & 145,00 & 36,00 & 13,40 & 67,10 \\
\hline $\mathbf{P 2}$ & 7,10 & 303,00 & 0,35 & 18,00 & 337,60 & 215,00 & 22,00 & 38,90 & 6,00 \\
\hline P3 & 4,00 & 190,00 & 0,30 & 0,00 & 88,30 & 15,00 & 0,00 & 3,70 & 23,30 \\
\hline P4 & 5,20 & 187,00 & 0,60 & 0,00 & 103,50 & 45,00 & 4,00 & 8,50 & 9,00 \\
\hline P5 & 5,20 & 312,00 & 0,35 & 0,00 & 171,00 & 100,00 & 16,00 & 14,60 & 7,50 \\
\hline P6 & 5,40 & 201,00 & 1,15 & 5,40 & 156,80 & 65,00 & 12,00 & 8,50 & 9,00 \\
\hline P7 & 4,70 & 954,00 & 0,30 & 0,00 & 388,80 & 250,00 & 24,00 & 46,20 & 28,20 \\
\hline P8 & 5,90 & 1276,00 & 3,85 & 16,20 & 600,50 & 280,00 & 50,00 & 37,70 & 78,50 \\
\hline P9 & 6,20 & 707,00 & 0,30 & 0,00 & 312,90 & 180,00 & 10,00 & 37,70 & 29,90 \\
\hline P10 & 3,80 & 260,00 & 0,60 & 1,80 & 117,40 & 20,00 & 0,00 & 4,90 & 23,30 \\
\hline P11 & 3,40 & 727,00 & 0,30 & 0,00 & 258,50 & 85,00 & 8,00 & 15,80 & 56,00 \\
\hline P12 & 5,20 & 820,00 & 0,45 & 1,80 & 360,50 & 220,00 & 30,00 & 35,20 & 26,60 \\
\hline P13 & 6,00 & 476,00 & 0,40 & 0,00 & 311,00 & 145,00 & 30,00 & 17,00 & 21,70 \\
\hline Poços & $\mathbf{K}$ & $\mathrm{HCO}_{3}$ & $\mathrm{CO}_{3}$ & $\mathrm{Cl}$ & $\mathrm{SO}_{4}$ & Fe & $\mathrm{N}-\mathrm{NH}_{4}$ & $\mathrm{~N}-\mathrm{NO}_{2}$ & $\mathrm{~N}-\mathrm{NO}_{3}$ \\
\hline P1 & 6,20 & 274,60 & 0,00 & 24,00 & 87,90 & 0,02 & 0,11 & 0,43 & 0,00 \\
\hline P2 & 8,30 & 242,30 & 0,00 & 12,00 & 4,50 & 0,00 & 2,59 & 1,05 & 0,00 \\
\hline $\mathbf{P 3}$ & 6,20 & 8,10 & 0,00 & 26,40 & 0,20 & 0,00 & 0,00 & 1,19 & 19,20 \\
\hline P4 & 6,20 & 32,30 & 0,00 & 21,60 & 1,50 & 0,00 & 1,00 & 0,55 & 18,70 \\
\hline P5 & 8,30 & 48,50 & 0,00 & 48,10 & 0,10 & 0,00 & 2,81 & 1,15 & 24,00 \\
\hline P6 & 6,20 & 96,90 & 0,00 & 12,00 & 4,60 & 0,03 & 0,00 & 0,51 & 6,90 \\
\hline P7 & 21,50 & 32,30 & 0,00 & 120,20 & 0,10 & 0,00 & 1,03 & 0,87 & 114,50 \\
\hline P8 & 31,70 & 40,40 & 0,00 & 204,30 & 4,00 & 0,06 & 0,91 & 1,35 & 151,60 \\
\hline P9 & 19,70 & 16,20 & 0,00 & 101,00 & 0,10 & 0,00 & 1,11 & 1,19 & 96,10 \\
\hline P10 & 16,00 & 8,10 & 0,00 & 33,70 & 0,20 & 0,00 & 0,00 & 0,43 & 30,90 \\
\hline P11 & 12,20 & 0,00 & 0,00 & 84,10 & 1,80 & 0,00 & 1,32 & 1,32 & 78,00 \\
\hline P12 & 19,70 & 40,40 & 0,00 & 93,70 & 0,87 & 0,00 & 0,00 & 0,55 & 113,40 \\
\hline P13 & 8,30 & 145,40 & 0,00 & 43,30 & 10,00 & 0,00 & 0,00 & 0,63 & 34,80 \\
\hline
\end{tabular}

Legenda: $\mathrm{pH}$ - potencial hidrogeniônico; CE - condutividade elétrica $(\mu \mathrm{S} / \mathrm{cm}) ; \mathrm{STD}$ - sólidos totais dissolvidos (mg/L); DT Dureza total (mg/L de $\left.\mathrm{CaCO}_{3}\right)$; Ca - Cálcio (mg/L); Mg - Magnésio (mg/L); Na-Sódio (mg/L); K-Potássio (mg/L); HCO 3 Bicarbonato (mg/L); $\mathrm{CO}_{3}$-Carbonato (mg/L), Cl - Cloretos (mg/L); $\mathrm{SO}_{4}$ - Sulfato (mg/L); Fe-Ferro ((mg/L); N-NH - Amônia $(\mathrm{mg} / \mathrm{L}) ; \mathrm{N}-\mathrm{NO}_{2}-\mathrm{Nitrito}(\mathrm{mg} / \mathrm{L}) ; \mathrm{N}-\mathrm{NO}_{3}$ - Nitrato $(\mathrm{mg} / \mathrm{L})$.

Fonte: banco de dados da Companhia de Gestão dos Recursos Hídricos (COGERH).

Em função do conjunto de variáveis analisadas ( $\mathrm{pH}$ - potencial hidrogeniônico, $\mathrm{CE}$ - condutividade elétrica, turbidez, cor, STD - sólidos totais dissolvidos, dureza total, $\mathrm{Ca}^{++}, \mathrm{Mg}^{++}, \mathrm{Na}^{+}, \mathrm{K}^{+}$, $\mathrm{HCO}_{3}^{-}, \mathrm{CO}_{3}, \mathrm{Cl}^{-}, \mathrm{SO}_{4}, \mathrm{~N}-\mathrm{NH}_{4}, \mathrm{~N}-\mathrm{NO}_{2}, \mathrm{~N}-\mathrm{NO}_{3}$ e $\mathrm{Fe}$ ) foi utilizado o procedimento de análise multivariada, envolvendo análise fatorial pelo método das componentes principais e análise de agrupamento hierárquico. Segundo Brito et al. (2006), os fatores principais classificam as variáveis de maior participação em cada fator, definindo as que devem ser monitoradas, e assim, reduzindo custos com análises de variáveis de menor importância na qualidade das águas. 
Os fatores são calculados pela combinação linear das variáveis originais através do método da análise da componente principal (ACP), utilizandose a rotação VARIMAX normalizada. Neste procedimento os dados foram padronizados pelo método $\mathrm{Z}$ scores com o intuito de eliminar os efeitos produzidos pelas diferentes escalas e unidades. Para verificar a adequação dos dados à análise fatorial utilizou-se o índice KMO (KaiserMeyer-Olkin Measure of Sampling Adequacy) e o teste estatístico de esfericidade de Bartlett (Corrar et al., 2014).

Tabela 2 - Análises físico-químicas das águas subterrâneas de Juazeiro do Norte/CE (julho/2011).

\begin{tabular}{|c|c|c|c|c|c|c|c|c|c|}
\hline \multirow[b]{2}{*}{ Poços } & \multirow[b]{2}{*}{ pH } & \multirow[b]{2}{*}{$\mathbf{C E}$} & \multirow[b]{2}{*}{ Turbidez } & \multicolumn{6}{|c|}{ Período seco (julho/2011) } \\
\hline & & & & Cor & STD & DT & $\mathbf{C a}$ & Mg & $\mathbf{N a}$ \\
\hline P1 & 7,90 & 583,00 & 0,70 & 0,00 & 535,70 & 152,00 & 35,20 & 15,60 & 73,60 \\
\hline $\mathbf{P 2}$ & 7,83 & 387,00 & 0,50 & 0,00 & 363,70 & 180,00 & 30,40 & 25,30 & 15,60 \\
\hline $\mathbf{P 3}$ & 5,06 & 176,00 & 0,35 & 1,80 & 96,60 & 16,00 & 1,60 & 2,90 & 23,70 \\
\hline P4 & 6,30 & 181,00 & 0,55 & 0,00 & 137,70 & 64,00 & 12,80 & 7,80 & 11,70 \\
\hline P5 & 5,87 & 286,00 & 0,40 & 0,00 & 146,60 & 84,00 & 14,40 & 11,70 & 7,70 \\
\hline P6 & 6,50 & 179,00 & 1,25 & 0,00 & 162,70 & 80,00 & 14,40 & 10,70 & 10,70 \\
\hline P7 & 5,45 & 827,00 & 0,45 & 0,00 & 356,20 & 200,00 & 27,20 & 32,10 & 32,00 \\
\hline P8 & 6,20 & 1233,00 & 1,10 & 3,60 & 538,80 & 288,00 & 49,60 & 39,90 & 78,20 \\
\hline P9 & 5,11 & 700,00 & 0,45 & 0,00 & 301,20 & 160,00 & 11,20 & 32,10 & 29,90 \\
\hline P10 & 4,73 & 277,00 & 0,40 & 0,00 & 106,90 & 12,00 & 1,60 & 1,90 & 21,70 \\
\hline P11 & 4,01 & 551,00 & 0,30 & 0,00 & 221,20 & 76,00 & 4,80 & 15,60 & 42,50 \\
\hline P12 & 5,75 & 725,00 & 0,40 & 0,00 & 323,40 & 204,00 & 27,20 & 33,10 & 17,60 \\
\hline P13 & 6,74 & 418,00 & 0,40 & 0,00 & 317,90 & 188,00 & 52,80 & 13,60 & 21,70 \\
\hline Poços & $\mathbf{K}$ & $\mathrm{HCO}_{3}$ & $\mathrm{CO}_{3}$ & $\mathrm{Cl}$ & $\mathrm{SO}_{4}$ & Fe & $\mathrm{N}-\mathrm{NH}_{4}$ & $\mathrm{~N}-\mathrm{NO}_{2}$ & $\mathrm{~N}-\mathrm{NO}_{3}$ \\
\hline P1 & 6,40 & 281,10 & 0,00 & 28,80 & 94,80 & 0,07 & 0,00 & 0,22 & 0,00 \\
\hline $\mathbf{P 2}$ & 9,10 & 245,50 & 0,00 & 25,00 & 12,50 & 0,00 & 0,00 & 0,35 & 0,00 \\
\hline P3 & 9,10 & 12,90 & 0,00 & 32,70 & 0,50 & 0,00 & 0,00 & 0,22 & 13,00 \\
\hline P4 & 9,10 & 64,60 & 0,00 & 21,20 & 1,20 & 0,00 & 0,00 & 0,22 & 9,30 \\
\hline P5 & 8,20 & 48,50 & 0,00 & 44,20 & 0,30 & 0,23 & 0,00 & 0,31 & 11,10 \\
\hline P6 & 6,40 & 103,40 & 0,00 & 11,50 & 5,10 & 0,12 & 0,00 & 0,43 & 0,00 \\
\hline P7 & 25,30 & 42,00 & 0,00 & 121,10 & 3,10 & 0,00 & 0,00 & 0,26 & 73,10 \\
\hline P8 & 29,00 & 54,90 & 0,00 & 217,30 & 5,30 & 0,00 & 0,00 & 1,15 & 63,50 \\
\hline P9 & 29,00 & 32,30 & 0,00 & 103,80 & 0,10 & 0,00 & 0,00 & 0,83 & 61,90 \\
\hline P10 & 17,50 & 0,00 & 0,00 & 40,40 & 0,00 & 0,00 & 0,00 & 0,22 & 23,60 \\
\hline P11 & 17,50 & 0,00 & 0,00 & 84,60 & 0,90 & 0,00 & 0,05 & 0,67 & 54,60 \\
\hline P12 & 25,30 & 51,70 & 0,00 & 98,10 & 2,90 & 0,00 & 0,00 & 0,31 & 67,20 \\
\hline P13 & 9,10 & 161,50 & 0,00 & 38,50 & 11,10 & 0,00 & 0,00 & 0,39 & 9,30 \\
\hline
\end{tabular}

Legenda: $\mathrm{pH}$ - potencial hidrogeniônico; $\mathrm{CE}$ - condutividade elétrica $(\mu \mathrm{S} / \mathrm{cm})$; STD - sólidos totais dissolvidos (mg/L); DTDureza total (mg/L de $\left.\mathrm{CaCO}_{3}\right)$; Ca-Cálcio (mg/L); Mg-Magnésio (mg/L); Na-Sódio (mg/L); K-Potássio (mg/L); HCO 3 Bicarbonato (mg/L); $\mathrm{CO}_{3}$-Carbonato (mg/L), Cl-Cloretos (mg/L); $\mathrm{SO}_{4}$-Sulfato (mg/L); Fe-Ferro ((mg/L); N-NH$H_{4}-\mathrm{Amônia}$ $(\mathrm{mg} / \mathrm{L}) ; \mathrm{N}-\mathrm{NO}_{2}-\mathrm{Nitrito}(\mathrm{mg} / \mathrm{L}) ; \mathrm{N}^{-N_{3}}{ }_{3}$-Nitrato $(\mathrm{mg} / \mathrm{L})$.

Fonte: banco de dados da Companhia de Gestão dos Recursos Hídricos (COGERH).

Em seguida realizou-se a análise de agrupamento, objetivando agrupar amostras a partir do maior número de atributos explicados por um único fator da análise fatorial. Nessa etapa utilizou-se o método Ward's como critério hierárquico de agrupamento e a medida de similaridade utilizada foi a "distância euclidiana quadrada" (squared euclidean distance) (Hair et al., 2009).
A escolha deste critério se baseou na frequente empregabilidade do mesmo em estudos de qualidade da água (Yidana et al., 2008; Palácio et al., 2009; Fernandes et al., 2010; Salgado et al., 2011; Gomes \& Cavalcante, 2017).

O ponto de corte utilizado na análise de agrupamento foi definido a partir da proposta de Corrar et al. (2014), que consiste em 
examinar alguma medida de similaridade ou distância entre grupos a cada passo sucessivo, até quando os sucessivos valores entre os passos tiverem uma súbita elevação na diferença entre coeficientes, apresentada no cronograma de aglomeração.

\section{RESULTADOS E DISCUSSÕES}

A análise fatorial pelo método da componente principal inicialmente foi realizada com 18 variáveis físico-químicas $(\mathrm{pH}, \mathrm{CE}$, turbidez, cor, STD, dureza total, $\mathrm{Ca}^{++}, \mathrm{Mg}^{++}, \mathrm{Na}^{+}, \mathrm{K}^{+}, \mathrm{HCO}_{3}$, $\mathrm{CO}_{3}, \mathrm{Cl}^{-}, \mathrm{SO}_{4}, \mathrm{~N}-\mathrm{NH}_{4}, \mathrm{~N}-\mathrm{NO}_{2}, \mathrm{~N}-\mathrm{NO}_{3}$ e $\mathrm{Fe}$ ) em dois períodos distintos, janeiro (período chuvoso) e julho (período seco), onde foram necessárias três simulações para atingir um resultado satisfatório, levando em conta os critérios adotados para essa análise, reduzindo significativamente o número de variáveis na última simulação.

A simulação final resultou com 10 variáveis (CE, STD, dureza total, $\mathrm{Ca}^{2+}, \mathrm{Mg}^{2+}, \mathrm{K}^{+}, \mathrm{Cl}^{-}, \mathrm{N}-$ $\mathrm{NO}_{3}, \mathrm{HCO}_{3}$ e $\mathrm{pH}$ ) e apresentou dois fatores nos dois períodos analisados que descreveram adequadamente a variação dos dados. $\mathrm{O}$ valor de $K M O$ foi de 0,580 (janeiro) e 0,653 (julho) e os testes estatísticos de esfericidade de Bartlett mostraram-se significativos a $p<0,01$. A partir dos resultados obtidos nos períodos de janeiro e julho de 2011, observa-se que os dois fatores respondem conjuntamente por $89 \%$ e $93 \%$ da variância total acumulada. $\mathrm{O}$ fator 1 , nos respectivos períodos, potencializa as variáveis CE, STD, dureza total, $\mathrm{Ca}^{2+}, \mathrm{Mg}^{2+}, \mathrm{K}^{+}, \mathrm{Cl}^{-}$e N-
$\mathrm{NO}_{3}$, fortemente relacionadas com a salinidade e compostos nitrogenados nas águas, cujas cargas fatoriais são superiores a 0,678 , indicando que os mesmos são os mais significativos na caracterização da qualidade das águas na área de estudo (63 e 57\% da variância total do conjunto amostral em cada período). O fator 2 está definido pelas variáveis $\mathrm{HCO}_{3}$ e pH (Tabela 3).

As variáveis do Fator 1 (CE, STD, dureza total, $\mathrm{Ca}^{2+}, \mathrm{Mg}^{2+}, \mathrm{K}^{+}, \mathrm{Cl}^{-}$e $\mathrm{N}-\mathrm{NO}_{3}$ ) podem estar relacionados, conforme Brodnjak-Voncina et al. (2002), com o processo natural de intemperismo dos componentes geológicos do solo. Correlações semelhantes também foram encontradas por Palácio (2004) e Gomes et al. (2017).

As variáveis com maiores cargas fatoriais, nesse fator, foram CE $(0,946$ e 0,951), K (0,957 e 0,933$), \mathrm{Cl}(0,980$ e 0,960$)$ e $\mathrm{N}-\mathrm{NO}_{3}(0,983$ e $0,915)$, embora as demais variáveis também apresentem forte relação com esse fator, os dados elevados reforçam a hipótese da diluição de esgoto infiltrado nos aquíferos, tendo, consequentemente, grande correlação com a $\mathrm{CE}$, indicando a importância desse processo no aumento da salinidade das águas (Tabela 3).

Tabela 3 - Cargas fatoriais e variância explicada na análise fatorial das 10 variáveis, após a rotação pelo método VARIMAX.

\begin{tabular}{l|c|c|c|c}
\hline \multirow{2}{*}{ Variáveis } & \multicolumn{2}{|c|}{ Janeiro/2011 } & \multicolumn{2}{c}{ Julho/2011 } \\
\cline { 2 - 5 } & \multicolumn{2}{|c}{ Carga fatorial } & \multicolumn{2}{c}{ Carga fatorial } \\
\cline { 2 - 5 } pH & Fator 1 & Fator 2 & Fator 1 & Fator 2 \\
\hline CE & 0,009 & $\mathbf{0 , 8 6 9}$ & $-0,262$ & $\mathbf{0 , 9 3 7}$ \\
\hline STD & $\mathbf{0 , 9 4 6}$ & 0,211 & $\mathbf{0 , 9 5 1}$ & 0,251 \\
\hline Dureza total & $\mathbf{0 , 6 7 8}$ & 0,662 & $\mathbf{0 , 7 5 4}$ & 0,589 \\
\hline Ca & $\mathbf{0 , 7 4 8}$ & 0,624 & $\mathbf{0 , 7 5 2}$ & 0,632 \\
\hline Mg & $\mathbf{0 , 7 2 9}$ & 0,551 & $\mathbf{0 , 8 3 0}$ & 0,386 \\
\hline K & $\mathbf{0 , 7 3 6}$ & 0,450 & $\mathbf{0 , 8 8 8}$ & 0,328 \\
\hline HCO3 & $\mathbf{0 , 9 5 7}$ & $-0,035$ & $\mathbf{0 , 9 3 3}$ & $-0,265$ \\
\hline Cl & $-0,390$ & $\mathbf{0 , 8 8 8}$ & $-0,299$ & $\mathbf{0 , 9 2 1}$ \\
\hline N-NO3 & $\mathbf{0 , 9 8 0}$ & $-0,016$ & $\mathbf{0 , 9 6 0}$ & $-0,029$ \\
\hline Variância explicada pelo fator $(\%)$ & 63,036 & 26,143 & 57,577 & 35,242 \\
\hline Variância acumulada $(\%)$ & $\mathbf{0 , 9 8 3}$ & $-0,126$ & $\mathbf{0 , 9 1 5}$ & $-0,341$ \\
\hline
\end{tabular}

Legenda: pH-potencial hidrogeniônico; CE-condutividade elétrica $(\mu \mathrm{S} / \mathrm{cm}) ;$ STD-sólidos totais dissolvidos (mg/L); Dureza total (mg/L de $\left.\mathrm{CaCO}_{3}\right)$; Ca-Cálcio (mg/L); Mg-Magnésio (mg/L); K-Potássio (mg/L); $\mathrm{HCO}_{3}$-Bicarbonato (mg/L);

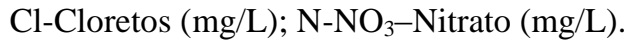


Em Juazeiro do Norte é comum o lançamento de efluentes não tratados no riacho dos Macacos (Figura 2) e rio da Batateira (riacho Salgadinho), introduzindo quantidades elevadas de carbono orgânico solúvel, nitrogênio, cloretos, potássio e magnésio nas águas superficiais, que por conexão são capazes de atingir os aquíferos. Segundo Lucena et al. (2004), ao atingir o aquífero, estes íons podem adquirir uma razoável mobilidade, capaz de alcançar eventualmente áreas que teoricamente seriam de baixo risco de contaminação por uma dessas atividades humanas.

Machado (2005) também observou uma forte correlação entre as concentrações de nitrato, cloreto e CE de amostras de águas subterrâneas coletadas nos municípios de Juazeiro do Norte e Crato. Segundo o autor, elevados valores de CE, nestas condições, pode ser um indicativo de contaminação por esgoto doméstico.

Santos et al. (2014) utilizaram o íon cloreto como traçador no estudo da conexão entre um riacho perenizado por esgoto e o aquífero, mostrando que o transporte de contaminante ocorre, predominantemente, na vertical, induzido pelo bombeamento dos poços e pela recarga do aquífero a partir do riacho dos Macacos (Figura 2). $\mathrm{O}$ fator $2\left(\mathrm{HCO}_{3} \mathrm{e} \mathrm{pH}\right)$ corresponde a $26 \mathrm{e}$ $35 \%$ de toda a variância do conjunto amostral nos dois períodos (Tabela 3). Este resultado indica a forte relação entre o $\mathrm{pH}$ e as concentrações de gás carbônico, bicarbonatos e carbonatos, corroborando com Pina (2012) e Gomes \& Cavalcante (2017). Segundo Neto (1966) apud Pina (2012), a maioria dos íons bicarbonatos $\left(\mathrm{HCO}_{3}{ }^{-}\right)$e carbonatos $\left(\mathrm{CO}_{3}^{-2}\right)$ em águas subterrâneas são derivados do $\mathrm{CO}_{2}$ na atmosfera e no solo, e de soluções de rochas carbonatadas.

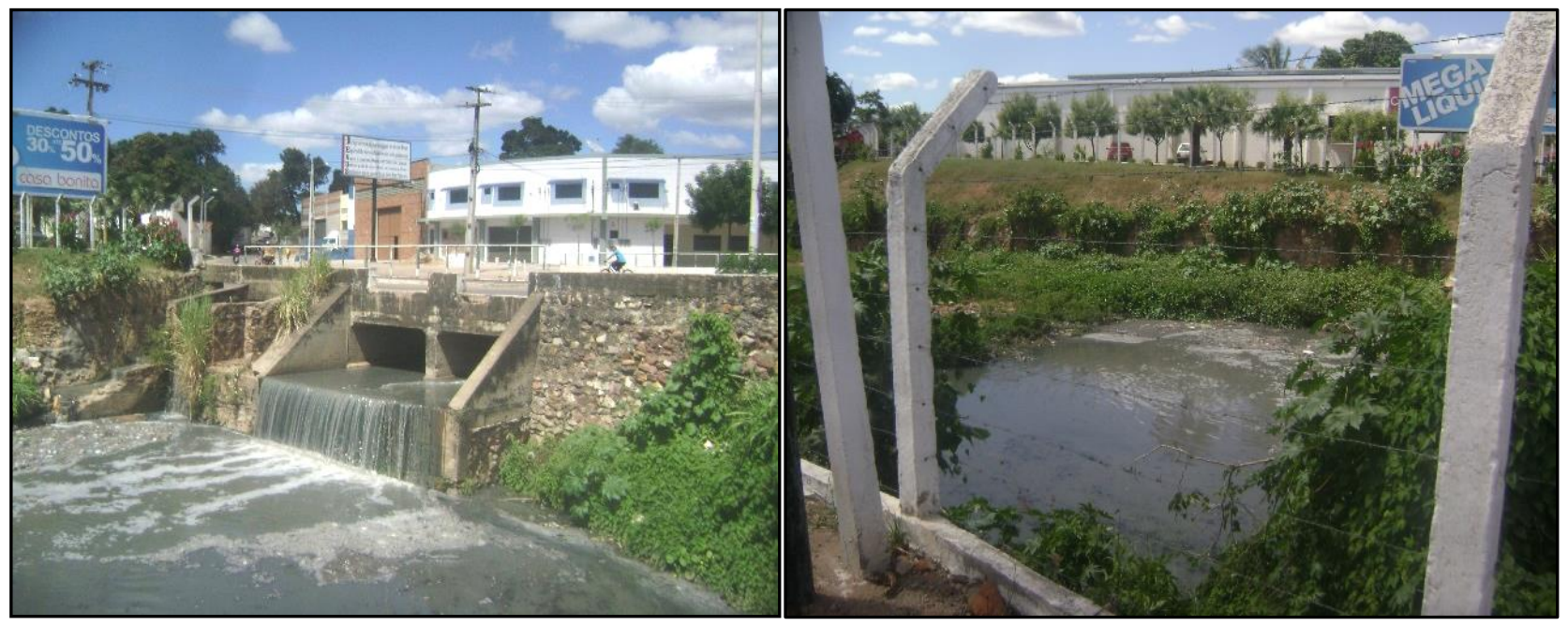

Figura 2 - Riacho dos Macacos, Juazeiro do Norte - Ceará. Coord. UTM: 9201219/466229 (setembro/2015).

Em função das maiores cargas fatoriais das variáveis que compõem os dois fatores, as 10 variáveis de qualidade das águas podem ser representadas por: Fator 1 - componente de salinidade (mineralização) e atividades antrópicas (contaminação com elemento nitrogenado) e Fator 2 - componente de alcalinidade. Essas variáveis são determinantes na caracterização da qualidade das águas subterrâneas. A análise de agrupamento foi realizada com o objetivo de avaliar a semelhança das águas subterrâneas na área de estudo, quanto à origem das mesmas.

Sendo assim quatro dendrogramas foram gerados para agrupar as amostras de águas similares para os Fatores 1 e 2 em cada período analisado. Os grupos foram definidos pelo ponto de corte dos dendrogramas, representados nas figuras por linhas vermelhas (Figuras 3 e 4). Os grupos definidos para o fator 1 (CE, STD, dureza total, $\mathrm{Ca}^{2+}, \mathrm{Mg}^{2+}, \mathrm{K}^{+}, \mathrm{Cl}^{-}$e N-NO$)_{3}$, nos períodos de chuva (janeiro/2011) e seco (julho/2011) (Figuras 3 e 4) são indicadores de sais e poluição de origem orgânica.

De acordo com as variáveis do fator $1(\mathrm{CE}$, STD, dureza total, $\mathrm{Ca}^{2+}, \mathrm{Mg}^{2+}, \mathrm{K}^{+}, \mathrm{Cl}^{-}$e N-NO 3 ) no período de chuva foram gerados 4 grupos similares (G1, G2, G3 e G4), composto por $38 \%$ (5 poços), $31 \%$ (4 poços), $23 \%$ (3 poços) e $8 \%$ (1 poço) das amostras analisadas nos Grupos 1, 2, 3 e 4 (Figura 3A), respectivamente.

Enquanto no período seco foram produzidos 3 grupos (G1, G2 e G3), composto por $38 \%$ (5 poços), $54 \%$ ( 7 poços) e $8 \%$ (1 poço) das amostras analisadas nos Grupos 1, 2 e 3 (Figura $4 \mathrm{C})$, respectivamente. 

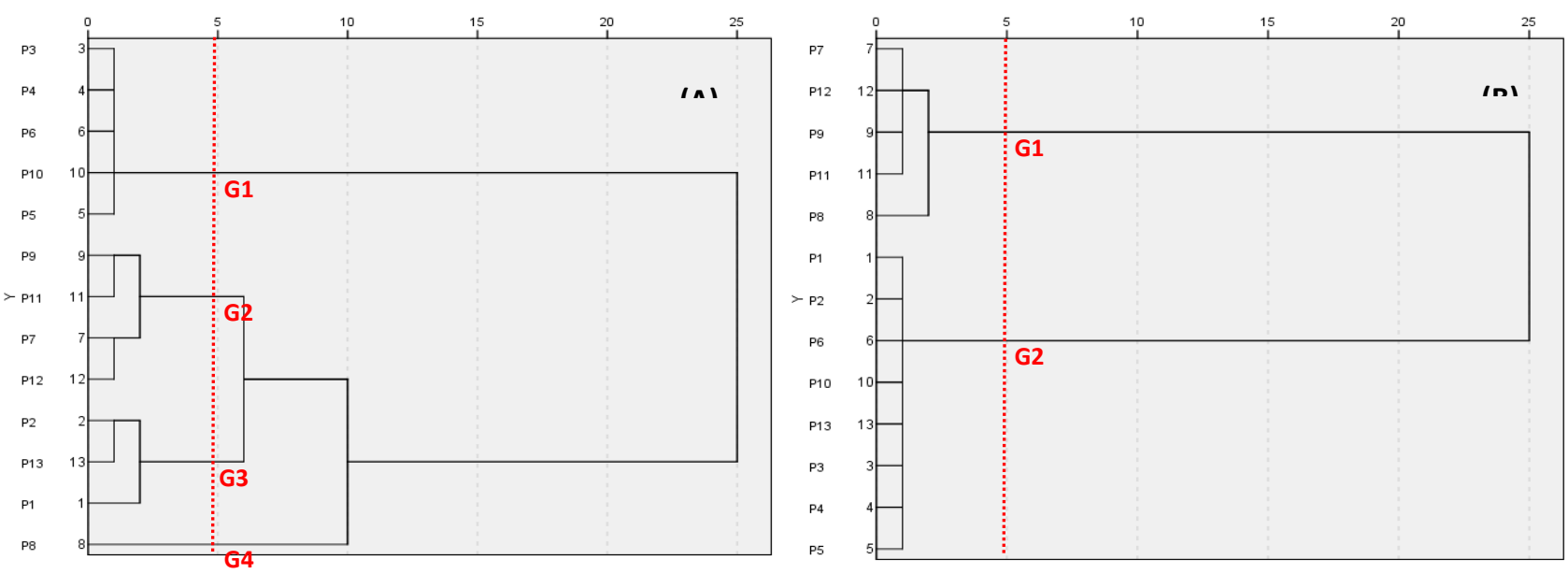

Figura 3 - Dendrogramas resultante da análise de agrupamento hierárquico das variáveis explicadas nos Fatores 1 (A) e 2 (B) no período de chuva (janeiro/2011).
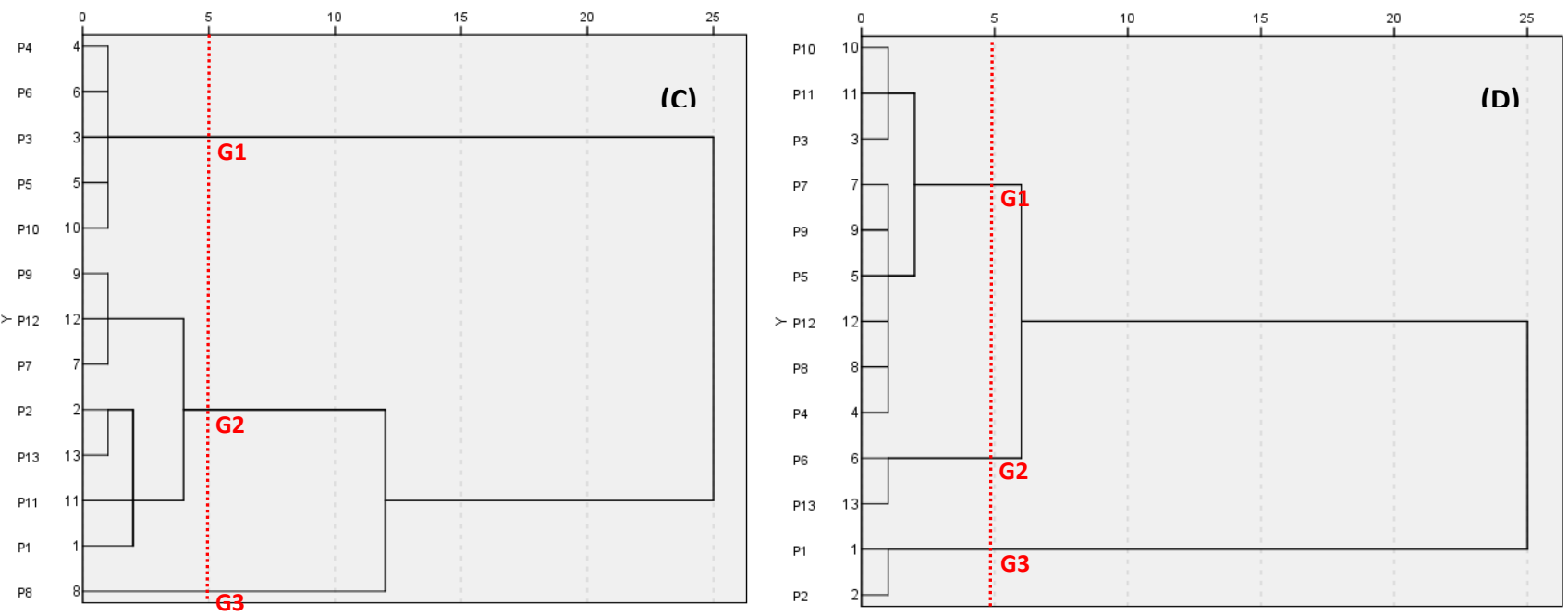

Figura 4 - Dendrogramas resultante da análise de agrupamento hierárquico das variáveis explicadas nos Fatores 1 (C) e 2 (D) no período seco (julho/2011).

O Grupo 1 (5 poços) apresentou as mesmas amostras (P3, P4, P5, P6 e P10) nos dois períodos analisados (chuva e seco), caracterizados por águas de baixas concentrações de sais e detém águas de melhor qualidade, exceto em relação ao elemento nitrogenado, com CE (média) entre 230 (chuva) e $220 \mu \mathrm{S} / \mathrm{cm}$ (seco).

Os Sólidos Totais Dissolvidos oscilaram de 127 a $130 \mathrm{mg} / \mathrm{L}$ (média), no período de chuva e seco, respectivamente, estando, todas as amostras dentro do limite permitido $(1000 \mathrm{mg} / \mathrm{L})$ para águas potáveis para consumo humano conforme a Portaria $n^{\circ}$ 2914/2011 do Ministério da Saúde (MS). A dureza total das amostras atingiu no máximo 100 (chuva) e $84 \mathrm{mg} / \mathrm{L}$ (seco), estando dentro do limite permitido $(500 \mathrm{mg} / \mathrm{L})$ pela Portaria vigente. A concentração de cloreto nas águas foi no máximo de 48 (chuva) e 44 mg/L (seco), estando também dentro do valor máximo permitido $(250 \mathrm{mg} / \mathrm{L})$.

Em relação aos íons cálcio, magnésio e potássio, a Portaria $\mathrm{n}^{\text {o }}$ 2914/2011 do MS (BRASIL, 2011) não estabelece um valor máximo permissível para suas concentrações. As concentrações médias de cálcio, magnésio e potássio no período de chuva, foram de 6,8 e 9 $\mathrm{mg} / \mathrm{L}$, enquanto no período seco foram de 9,7 e $10 \mathrm{mg} / \mathrm{L}$, respectivamente.

Das cinco (5) amostras de águas dos poços, quatro $(80 \%)$ e três $(60 \%)$ amostras no período de chuva e seco, respectivamente, não atendem aos padrões de potabilidade, quanto a concentração de nitrato $\left(10 \mathrm{mg} / \mathrm{L}\right.$ de $\left.\mathrm{N}-\mathrm{NO}_{3}{ }^{-}\right)$, variando de 7 a $31 \mathrm{mg} / \mathrm{L}$ (chuva) e 9 a $24 \mathrm{mg} / \mathrm{L}$ (seco) de $\mathrm{N}$-nitrato, refletindo o efeito das ações antrópicas na área, independente do período de 
análise. O poço P10 (108 m de profundidade) locado nas coberturas arenosas foi $o$ que apresentou o maior valor de nitrato na área, atingindo 31 (chuva) e $24 \mathrm{mg} / \mathrm{L}$ (seco).

Segundo Veríssimo (1999), esse litotipo tem como função principal realimentar os aquíferos subjacentes e situam-se, predominantemente, entre as cotas 440 a $480 \mathrm{~m}$.

O Grupo 2 nos dois períodos, é caracterizado por águas de salinidade intermediária e detém águas de melhor qualidade, exceto em relação ao elemento nitrogenado. No período de chuva (P7, P9, P11 e P12) a CE ficou entre 707 e $954 \mu \mathrm{S} / \mathrm{cm}$. Os Sólidos Totais Dissolvidos oscilaram de 259 a $389 \mathrm{mg} / \mathrm{L}$, estando, todas as amostras dentro do limite permitido (1000 $\mathrm{mg} / \mathrm{L})$ para águas potáveis para consumo humano conforme a Portaria $n^{\circ}$ 2914/2011 do Ministério da Saúde (MS). A dureza total das amostras atingiu no máximo $250 \mathrm{mg} / \mathrm{L}$, estando também dentro do limite permitido $(500 \mathrm{mg} / \mathrm{L})$ pela Portaria vigente.

A concentração de cloreto nas águas foi no máximo de $120 \mathrm{mg} / \mathrm{L}$, estando dentro do valor máximo permitido $(250 \mathrm{mg} / \mathrm{L})$. As concentrações médias de cálcio, magnésio e potássio foram de 18,34 e $18 \mathrm{mg} / \mathrm{L}$, respectivamente. Todas as amostras de águas dos poços (100\%) não atendem aos padrões de potabilidade, quanto a concentração de nitrato $\left(10 \mathrm{mg} / \mathrm{L}\right.$ de $\left.\mathrm{N}-\mathrm{NO}_{3}{ }^{-}\right)$, variando de 78 (P11 com profundidade de $70 \mathrm{~m}$ ) a $115 \mathrm{mg} / \mathrm{L}$ (P7 com profundidade de $84 \mathrm{~m}$ ) de $\mathrm{N}$-nitrato.

No período seco permanecem agrupados nesse grupo os 4 poços (P7, P9, P11 e P12) do período de chuva e mais 3 poços (P1, P2 e P13) passam a compor esse grupo. A CE variou $387 \mathrm{e}$ $827 \mu \mathrm{S} / \mathrm{cm}$. Os Sólidos Totais Dissolvidos oscilaram de 221 a $536 \mathrm{mg} / \mathrm{L}$, estando, todas as amostras dentro do limite permitido (1000 mg/L) para águas potáveis para consumo humano conforme a Portaria no 2914/2011 do MS. A dureza total das amostras atingiu no máximo 204 $\mathrm{mg} / \mathrm{L}$, estando também dentro do limite permitido $(500 \mathrm{mg} / \mathrm{L})$ pela Portaria vigente.

A concentração de cloreto nas águas foi no máximo de $121 \mathrm{mg} / \mathrm{L}$, estando dentro do valor máximo permitido $(250 \mathrm{mg} / \mathrm{L})$. As concentrações médias de cálcio, magnésio e potássio foram de 27, 24 e $17 \mathrm{mg} / \mathrm{L}$, respectivamente. Das 7 amostras de águas dos poços, $57 \%$ não atendem aos padrões de potabilidade, quanto a concentração de nitrato $\left(10 \mathrm{mg} / \mathrm{L}\right.$ de $\left.\mathrm{N}-\mathrm{NO}_{3}{ }^{-}\right)$, variando de 9 (P13 com profundidade de $140 \mathrm{~m}$ ) a $73 \mathrm{mg} / \mathrm{L}$ (P7 com profundidade de $84 \mathrm{~m}$ ) de Nnitrato.

O Grupo 3 no período de chuva ( 3 poços - P1, P2 e P13) é caracterizado por águas de salinidade baixa a média, com CE entre 303 e $610 \mu \mathrm{S} / \mathrm{cm}$. Os Sólidos Totais Dissolvidos oscilaram de 311 a $510 \mathrm{mg} / \mathrm{L}$, estando, todas as amostras dentro do limite permitido (1000 mg/L) para águas potáveis para consumo humano conforme a Portaria $\mathrm{n}^{\circ}$ 2914/2011 do MS. A dureza total das amostras atingiu no máximo $215 \mathrm{mg} / \mathrm{L}$, estando dentro do limite permitido $(500 \mathrm{mg} / \mathrm{L})$ pela Portaria vigente.

A concentração de cloreto nas águas foi no máximo de $43 \mathrm{mg} / \mathrm{L}$, estando também dentro do valor máximo permitido $(250 \mathrm{mg} / \mathrm{L})$. As concentrações médias de cálcio, magnésio e potássio foram de 29,23 e $8 \mathrm{mg} / \mathrm{L}$, respectivamente. Das três amostras de águas dos poços, apenas uma (P13 com $140 \mathrm{~m}$ de profundidade) não atende aos padrões de potabilidade, quanto a concentração de nitrato $\left(10 \mathrm{mg} / \mathrm{L}\right.$ de $\left.\mathrm{N}-\mathrm{NO}_{3}{ }^{-}\right)$, atingindo $35 \mathrm{mg} / \mathrm{L}$ de $\mathrm{N}$ nitrato.

No período seco, representado por apenas 1 poço (P8 - profundidade de $8 \mathrm{~m}$ ), o grupo 3 é caracterizado por água de salinidade alta, com CE e STD de $1233 \mu \mathrm{S} / \mathrm{cm}$ e $539 \mathrm{mg} / \mathrm{L}$, respectivamente, estando, a amostra dentro do limite permitido (1000 mg/L de STD) para águas potáveis para consumo humano conforme a Portaria n ${ }^{\circ}$ 2914/2011 do MS.

A dureza total da amostra atingiu $288 \mathrm{mg} / \mathrm{L}$, estando também dentro do limite permitido (500 $\mathrm{mg} / \mathrm{L}$ ) pela Portaria vigente. A concentração de cloreto foi de $217 \mathrm{mg} / \mathrm{L}$, estando dentro do valor máximo permitido $(250 \mathrm{mg} / \mathrm{L})$. As concentrações de cálcio, magnésio e potássio foram de 50, 40 e $29 \mathrm{mg} / \mathrm{L}$, respectivamente. A concentração de nitrato nas águas do poço P8 (captando água dos depósitos aluvionares) foi de $64 \mathrm{mg} / \mathrm{L}$ de $\mathrm{N}-\mathrm{NO}_{3}$, também não atendendo aos padrões de potabilidade, quanto a concentração de nitrato (10 mg/L de $\mathrm{N}-\mathrm{NO}_{3}{ }^{-}$).

O Grupo 4 somente ocorreu no período de chuva, representado pelo poço P8 (profundidade de $8 \mathrm{~m}$ ), composto por alta concentração de sais e qualidade da água inferior aos demais grupos, com CE e STD de $1276 \mu \mathrm{S} / \mathrm{cm}$ e $601 \mathrm{mg} / \mathrm{L}$, respectivamente, estando, a amostra dentro do 
limite permitido (1000 mg/L de STD) para águas potáveis para consumo humano conforme a Portaria n ${ }^{\circ}$ 2914/2011 do Ministério da Saúde.

A dureza total da amostra é de $280 \mathrm{mg} / \mathrm{L}$, estando dentro do limite permitido $(500 \mathrm{mg} / \mathrm{L})$ pela Portaria vigente. A concentração de cloreto foi de $204 \mathrm{mg} / \mathrm{L}$, estando também dentro valor máximo permitido $(250 \mathrm{mg} / \mathrm{L})$. As concentrações de cálcio, magnésio e potássio foram de 50, 38 e $32 \mathrm{mg} / \mathrm{L}$, respectivamente. O nitrato atingiu 152 $\mathrm{mg} / \mathrm{L}$, não atendendo aos padrões de potabilidade, quanto a concentração de nitrato $\left(10 \mathrm{mg} / \mathrm{L}\right.$ de $\left.\mathrm{N}-\mathrm{NO}_{3}{ }^{-}\right)$.

É comum na área a construção de poços rasos (profundidade até $20 \mathrm{~m}$ ) para serem utilizados na irrigação de cana-de-açúcar nestas aluviões.

Os grupos definidos para o fator $2(\mathrm{pH}$ e $\mathrm{HCO}_{3}$ ), nos períodos de chuva (janeiro/2011) e seco (julho/2011) (Figuras 3 e 4) são indicadores de alcalinidade.De acordo com as variáveis do

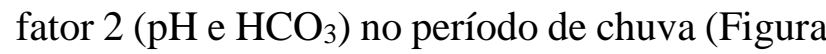
3B) foram gerados 2 grupos similares ( $\mathrm{G} 1$ e G2), composto por $38 \%$ ( 5 poços) e $62 \%$ ( 8 poços) das amostras analisadas nos Grupos 1 e 2, respectivamente. Enquanto no período seco (Figura 4B) foram produzidos 3 grupos (G1, G2 e G3), composto por $70 \%$ (9 poços), $15 \%$ (2 poços) e $15 \%$ ( 2 poços) das amostras analisadas nos Grupos 1, 2 e 3, respectivamente.

Os poços que compõem o Grupo 1 (P7, P8, P9, P11 e P12) no período de chuva, apresentaram uma maior similaridade das variáveis investigadas e qualidade das águas inferior em relação ao Grupo 2. São caracterizadas por águas ácidas $(100 \%$ das amostras), variando de 3,4 a 6,2 , onde apenas 1 amostra de água está dentro do limite permitido $\left(6,0\right.$ a 9,5) pela Portaria $n^{\circ} 2914 / 2011$ do Ministério da Saúde.

Os baixos valores $(<6)$ de $\mathrm{pH}$ encontrados nas águas desses poços estão associados provavelmente ao meio geológico, e pela introdução de águas recentes no aquífero, com concentrações mais elevadas de $\mathrm{CO}_{2}$, promovendo a diminuição do $\mathrm{pH}$ e aumentando, assim, a acidez das águas subterrâneas. Apresentaram as menores concentrações de bicarbonato (máximo de $40 \mathrm{mg} / \mathrm{L}$ ) nas águas em relação ao Grupo 2.

No período seco, o grupo 1, composto por nove amostras (P3, P4, P5, P7, P8, P9, P10, P11 e P12) é representado também por águas ácidas
(100\% das amostras), variando de 4,0 a 6,3, onde apenas 2 amostras de água estão dentro do limite permitido $\left(6,0\right.$ a 9,5) pela Portaria $n^{\circ} 2914 / 2011$ do Ministério da Saúde. Segundo Hounslow (1995), a principal razão dos valores de $\mathrm{pH}$ da água serem inferiores a 6 é a quantidade significativa de minerais de argila; que dissolve e libera sílica e alumínio anormalmente altos para a água. Além da alcalinidade, o pH é essencialmente uma função do gás carbônico dissolvido $\left(\mathrm{CO}_{2}\right)$ e dos ácidos orgânicos disponíveis nos solos que aumentam a acidez das águas subterrâneas e a capacidade de dissolver Fe e Mg.

Apresentaram também baixas concentrações de bicarbonato (máximo de $65 \mathrm{mg} / \mathrm{L}$ ) nas águas. De acordo com Silva \& Bonotto (2006), os menores valores de bicarbonato indicam áreas de recarga e cresce de acordo com o sentido do fluxo subterrâneo.

O Grupo 2 (período de chuva) constituído por 8 poços (P1, P2, P3, P4, P5, P6, P10 e P13) apresentaram uma maior heterogeneidade quando comparado ao primeiro grupo. Há predominância também de águas ácidas $(87 \%$ das amostras). São águas com $\mathrm{pH}$, variando de 3,8 a 7,1, onde apenas duas amostras analisadas estão dentro do limite permitido $(6,0$ a 9,5$)$ pela Portaria $n^{\circ}$ 2914/2011 do Ministério da Saúde para águas de consumo humano.

Segundo Hounslow (1995), os valores mais elevados de $\mathrm{pH}$ geralmente se encontram em águas com predominância dos íons $\mathrm{Na}^{+} \mathrm{e} \mathrm{Ca}^{++}$ou em águas ricas em bicarbonatos; as águas poluídas geralmente são mais ácidas. Apresentaram as maiores concentrações de bicarbonato, com média de $107 \mathrm{mg} / \mathrm{L}$. Enquanto no período seco, o grupo 2, composto por duas amostras (P6 e P13) é representado também por águas ácidas, variando de 6,5 a 6,7, estando as amostras de água dentro do limite permitido $(6,0$ a 9,5) pela Portaria $n^{\circ} 2914 / 2011$ do Ministério da Saúde. As concentrações de bicarbonato (103 a $162 \mathrm{mg} / \mathrm{L}$ ) nas águas são maiores em relação ao Grupo 1 e inferiores ao Grupo 3.

O Grupo 3 somente ocorreu no período seco, representado pelos poços P1 (profundidade de $110 \mathrm{~m}$ ) e P2 (profundidade de $80 \mathrm{~m}$ ), onde são caracterizados por águas de qualidade superiores aos grupos anteriores. São águas básicas (alcalinas), variando de 7,9 a 7,8, estando dentro do limite permitido $(6,0$ a 9,5$)$ pela Portaria $n^{\circ}$ 
2914/2011 do Ministério da Saúde. Em consequência dos valores de $\mathrm{pH}$, as amostras apresentaram as maiores concentrações de bicarbonato (246 a $281 \mathrm{mg} / \mathrm{L}$ ) nas águas em relação aos Grupos 1 e 2.

Segundo Silva \& Bonotto (2006), em águas que contêm elevadas concentrações de íons de bicarbonato, há tendência de precipitação do cálcio e do magnésio sob a forma de carbonatos reduzindo, então, a concentração destes elementos na solução do solo e, consequentemente, aumentando a proporção de sódio. Esses poços estão locados na formação Brejo Santo (considerado como um aquiclude), subjacente a formação Rio da Batateira (aquífero), aflorando somente em pequena faixa descontínua ao norte do município de Juazeiro do Norte.

Funciona como camada selante da formação Mauriti e, devido à falta de dados locais, admitese espessuras em torno de $300 \mathrm{~m}$ (Mont'alverne et al., 1996) sendo designada como uma sequência de folhelhos, argilitos e arenitos.

A partir destes resultados pode-se observar que as características das águas estão fortemente relacionadas com a formação geológica dos poços estudados.

Portanto, medidas de monitoramento podem ser realizadas com base nos elementos hidroquímicos determinantes da qualidade das águas (CE, STD, dureza total, $\mathrm{Ca}^{2+}, \mathrm{Mg}^{2+}, \mathrm{K}^{+}, \mathrm{Cl}^{-}$ , $\mathrm{pH}, \mathrm{HCO}_{3}$ e $\mathrm{N}-\mathrm{NO}_{3}$ ) identificados pela análise fatorial. Estas medidas podem ser voltadas para conservação dos solos e melhor uso das águas subterrâneas do município de Juazeiro do Norte.

Gomes (2013) corrobora que a composição físico-química das águas subterrâneas depende, inicialmente, da composição das águas de recarga (pluviometria, águas superficiais) e, em seguida, de sua evolução química, influenciada diretamente pela interação água-rocha representada pela percolação da água através dos poros e/ou fraturas das camadas geológicas.

\section{CONSIDERAÇÕES FINAIS}

A aplicação da análise fatorial foi fundamental para subsidiar o monitoramento de qualidade das águas subterrâneas do município de Juazeiro do Norte, pois permitiu reduzir o número de variáveis à serem monitoradas de dezoito ( $\mathrm{pH}, \mathrm{CE}$, turbidez, cor, STD, dureza total, $\mathrm{Ca}^{++}, \mathrm{Mg}^{++}, \mathrm{Na}^{+}, \mathrm{K}^{+}, \mathrm{HCO}_{3}{ }^{-}, \mathrm{CO}_{3}, \mathrm{Cl}^{-}, \mathrm{SO}_{4}, \mathrm{~N}-$ $\mathrm{NH}_{4}, \mathrm{~N}-\mathrm{NO}_{2}, \mathrm{~N}-\mathrm{NO}_{3}$ e $\mathrm{Fe}$ ) para se considerar principalmente as sete variáveis relacionadas com a salinidade (CE, STD, dureza total, $\mathrm{Ca}^{2+}$, $\left.\mathrm{Mg}^{2+}, \mathrm{K}^{+}, \mathrm{Cl}^{-}\right)$, duas relacionadas à alcalinidade $\left(\mathrm{pH}\right.$ e $\left.\mathrm{HCO}_{3}\right)$ e os compostos nitrogenados $(\mathrm{N}-$ $\mathrm{NO}_{3}$ ). No geral, não ocorreram variações significativas nos dois períodos de análise da água (janeiro e julho), resultando, assim a redução dos custos, pela diminuição da quantidade de variáveis analisadas, como também, redução de tempo.

Procedimentos de agrupar as amostras de águas similares (originada da formação geológica e/ou atividade orgânica) em função das variáveis que definem a qualidade das águas facilitam o processo de gestão de recursos hídricos, principalmente nas atividades de monitoramento e de utilização das águas na área de estudo.

\section{REFERÊNCIAS}

BRASIL. Portaria no 2914 de 12 de dezembro de 2011 do Ministério da Saúde. Dispõe sobre os procedimentos de controle e vigilância da qualidade da água para consumo humano e seu padrão de potabilidade. Brasília, 34 p., 2011.

BRITO, L.T.L.; SILVA, A.S.; SRINIVASAN, V.S.; GALVÃO, C.O. Uso da análise multivariada para subsidiar no monitoramento da qualidade das águas da bacia hidrográfica do Salitre. In: CONGRESSO BRASILEIRO DE ENGENHARIA AGRÍCOLA, 2004, São Pedro. Anais...São Pedro. Sociedade Brasileira de Engenharia Agrícola. 2004.

BRITO, L.T.L.; SILVA, A.S.; SRINIVASAN, V.S.; GALVÃO, C.O.; GHEYI, H.R. Uso de análise multivariada na classificação das fontes hídricas subterrâneas na bacia hidrográfica do Salitre. Revista Engenharia Agrícola, v. 26, n. 1, p. 36-44, 2006.

BRODNJAK-VONCINA, D.; DOBČNIK, D.; DOBČNIK, D; NOVIČ, M, ZUPAN, J. Chemometrics characterisation of the quality of river water. Analytica Chimica Acta, v. 462, n. 1, p. 87-100, 2002.

CLOUTIER, V.; LEFEBVRE, R.; THERRIEN, R.; SAVARD,
M.M. Multivariate statistical analysis of geochemical data as indicative of the hydrogeo chemical evolution of groundwater in a sedimentary rock aquifer system. Journal of Hydrology, v. 353, p. 294-313, 2008.

COGERH-COMPANHIA DE GESTÃO DOS RECURSOS HÍDRICOS. Plano de monitoramento e gestão dos aquíferos da Bacia do Araripe. Relatório Final. 137 p., 2009.

CORRAR, L.J.; PAULO, E.; FILHO, J.M.D. (Coords). Análise Multivariada: para os Cursos de Administração, Ciências Contábeis e Economia. São Paulo: Atlas, 541 p., 2014.

CPRM-COMPANHIA DE PESQUISA DE RECURSOS MINERAIS. Atlas dos recursos hídricos subterrâneos do Ceará-Programa de recenseamento de fontes de abastecimento por águas subterrâneas do Ceará-Diagnóstico Municipal de Juazeiro do Norte. Fortaleza, 5 p., 2000.

FERNANDES, F.B.P.; ANDRADE, E.M.; FONTENELE, S. B.; MEIRELES, A.C.M.; RIBEIRO, J.A. Análise de agrupamento como suporte à gestão qualitativa da água subterrânea no semiárido cearense. Revista Agro@mbiente, v. 4, n. 2, p. 86$95,2010$. 
GOMES, M.C.R. Análise Situacional Qualitativa Sobre as Águas Subterrâneas de Fortaleza, Ceará/Brasil como Subsídio à Gestão dos Recursos Hídricos, 2013. 213 p. Tese (Doutorado em Geologia) - Universidade Federal do Ceará

GOMES, M.C.R. \& CAVALCANTE, I.N. Aplicação da análise estatística multivariada no estudo da qualidade da água subterrânea. Revista Águas Subterrâneas, v. 31, n.1, p. 134 149, 2017.

GOMES, M.C.R.; MENDONÇA, L.A.R.; LIMA, M.G.S.; ARAUUJ, A.O. Similaridade de atributos físico-químicos com uso de análise multivariada aplicada à gestão qualitativa de águas subterrâneas. Geociências, v. 36, n. 2, p. 325 - 337, 2017.

HAIR JR, J.F.; BLACK, W.C; BABIN, B.J.; ANDERSON, R.E.; TATHAM, R.L. Análise multivariada de dados. Porto Alegre, Bookman, 688 p., 2009.

HOUNSLOW, A.W. Water quality data: analysis and interpretation. Lewis Publishers New York. Boca Raton, 395 p., 1995.

IBGE-INSTITUTO BRASILEIRO DE GEOGRAFIA E ESTATÍSTICA. Censo geográfico 2010. Disp. em: htt://www.ibge.gov.br/cidadesat/topwindou.htm. Acessado em: 08 set2015.

IPECE-INSTITUTO DE PESQUISA E ESTRATÉGIA ECONÔMICA DO CEARÁ. Perfil Básico Municipal. Disponível em: htt://www.ipece.ce.gov.br. Acessado em: 06set 2015.

LUCENA, L.R.F.; ROSA FILHO, E.F. BITTENCOURT, A.V.L.; MONTAÑO, J.X. A migração de constituintes iônicos no aquífero Barreiras na região sul de Natal-RN, decorrente do quadro estrutural local - uma hipótese de trabalho. Revista Latino-Americana de Hidrogeologia, v. 4, n. 4, p. 9-16, 2004.

MACHADO, C.J.F. Modelagem geoquímica e de fluxos do sistema aquífero subterrâneo na Bacia Sedimentar do Araripe. Fortaleza, 2005. 159 p. Tese (Doutorado em Física) Universidade Federal do Ceará.

MONT"ALVERNE, A.A.F.; PONTE, F.C.; COSTA, W.C.; DANTAS, J.R.A; LOPES, C.F.; MELO JUNIOR, A.H.; PONTE, J.S.A; FILGUEIRA, J.B.M.; SOUZA, S.R.; SILVA, E.C.C. Projeto Avaliação hidrogeológica da bacia sedimentar do Araripe. Ministério das Minas e Energia. Departamento Nacional da Produção Mineral. Programa Nacional de Estudos dos Distritos Mineiros. - Fase I, Recife, 100 p., 1996.
PALÁCIO, H.A.Q. Índice de qualidade das águas na parte baixa da baciahidrográfica do rio Trussu, Ceará. Fortaleza, 2004. 96 p. Dissertação (Mestrado em Irrigação e Drenagem) Universidade Federal do Ceará.

PALÁCIO, H.A.Q.; ANDRADE, E.M.; LOPES, F.B.; ALEXANDRE, D.M.B.; ARRAES, F.D.D. Salinidade da qualidade das águas superficiais da bacia do Curu, Ceará. Ciência Rural, v. 39, n. 9, p. 2494-2500, 2009.

PINA, A.P.S.A. Fundamentos hidrogeoquímicos aplicados na Bacia Hidrográfica de Santa Cruz, Ilha de Santiago - Cabo Verde, como instrumento para a gestão de recursos hídricos. Fortaleza, 2012. 150 p. Dissertação (Mestrado em Geologia) - Universidade Federal do Ceará.

SALGADO, E.V.; ANDRADE, E.M., FONTENELE, S.B., MEIRELES, A.C.M. Similaridade das variáveis hidroquímicas com o uso da análise multivariada, na Bacia do Salgado, Ceará. Revista Caatinga, v. 24, n. 3, p. 158-166, 2011.

SANTOS, M.R.P.; SANTIAGO, M.F.; MENDONÇA, L.A.R.; FRISCHKORN, H.; MENDES FILHO, J. Modelagem do transporte de cloreto proveniente de esgoto urbano em um aquífero sedimentar usando MT3D: o caso da bateria de poços de Juazeiro do Norte (CE). Engenharia Sanitária e Ambiental, v. 19, n. 3, p. 283-292, 2014.

SILVA, M.L. \& BONOTTO, D.M. Hidroquímica elementar e dos isótopos de urânio em águas subterrâneas da formação Alter do Chão, Manaus (AM). Revista Brasileira de Geociência, v. 36, n.3, p. 437-448, 2006

VERÍSSIMO, L.S. A importância das Águas Subterrâneas para o Desenvolvimento Socioeconômico do Eixo CRAJUBAR, Cariri Ocidental - Estado do Ceará. Fortaleza, 1999. 140 p. Dissertação (Mestrado em Geologia) Universidade Federal do Ceará.

YIDANA, S.M.; OPHORI, D.; BANOENG-YAKUBO, B. A multivariate statistical analysis of surface water chemistry data. The Ankobra basin, Ghana. Journal of Environmental Management, n. 86, p. 80-87, 2008.

Submetido em 25 de fevereiro de 2018 Aceito em 4 de setembro de 2018 